\title{
Commerce, conflits et justice : les marchands portugais en Flandre à la fin du Moyen Âge
}

Flávio Miranda

\section{OpenEdition}

\section{Journals}

Édition électronique

URL : http://journals.openedition.org/abpo/1018

DOI : $10.4000 / a b p o .1018$

ISBN : 978-2-7535-1517-8

ISSN : 2108-6443

Éditeur

Presses universitaires de Rennes

Édition imprimée

Date de publication : 20 avril 2010

Pagination : 193-208

ISBN : 978-2-7535-1146-0

ISSN : 0399-0826

\section{Référence électronique}

Flávio Miranda, «Commerce, conflits et justice : les marchands portugais en Flandre à la fin du Moyen Âge », Annales de Bretagne et des Pays de l'Ouest [En ligne], 117-1 | 2010, mis en ligne le 20 avril 2012, consulté le 02 mai 2019. URL : http://journals.openedition.org/abpo/1018 ; DOI : 10.4000/abpo.1018

Ce document a été généré automatiquement le 2 mai 2019.

(c) Presses universitaires de Rennes 


\title{
Commerce, conflits et justice : les marchands portugais en Flandre à la fin du Moyen Âge ${ }^{1}$
}

\author{
Flávio Miranda
}

1 Le comté de Flandre a été pendant le Bas Moyen Âge un paradis pour les affaires et le commerce, avec des villes prospères qui ont bénéficié d'une diversité sociale reposant sur un équilibre entre noblesse, clergé, petits propriétaires de terres, agriculteurs, artisans et marchands ${ }^{2}$. Au-delà des conditions naturelles d'accès, qui ont subi des transformations au cours des siècles ${ }^{3}$, la ville de Bruges offrait des structures financières uniques: un système bancaire consolidé et la première bourse d'Europe. Le Conseil de Flandre disposait d'une jurisprudence propre en matière de commerce international, de mesures effectives et de procédures efficaces pour une rapide résolution des conflits. Le vaste ensemble de documents légaux, qui a survécu à ce jour, pourra être un reflet de ces caractéristiques. Très tôt les routes du commerce ont lié le sud et l'ouest de l'Europe à la Flandre, véritable terre promise ${ }^{4}$. Les Italiens de Gênes, sont arrivés les premiers, à la fin du XIII ${ }^{e}$ siècle ${ }^{5}$, suivis par les Vénitiens, les Catalans, les Castillans, enfin les Portugais, qui ont bénéficié de surcroît des bons rapports politiques entre le Portugal et la Flandre ${ }^{6}$. Bénéficiant des traités de liberté de commerce, de la protection offerte et de la demande de produits traditionnels de la péninsule Ibérique (de l'huile et du vin, des figues et des raisins secs, des fourrures et des cuirs, et plus tard le sucre), les Portugais ont établi une Nation à Bruges. Mais, en le faisant, auraient-ils cessé d'être des étrangers dans un pays avec une langue et des habitudes différentes?

2 Le propos de cet article est de comprendre l'activité en Flandre des marchands portugais - en tant que marchands étrangers - en analysant les rythmes du commerce et les conflits qui en résultaient, les mécanismes de gestion du risque et le rapport institutionnel avec la justice. Le marchand portugais éprouvait-il le besoin de se protéger et si oui, de qui ? Quels ont été les problèmes entre les marchands portugais et les marchands des autres pays? Quels étaient les mécanismes de protection à leur disposition? Dans quelle mesure 
les marchands portugais se sentaient-ils des étrangers en Flandre? Quel fut leur niveau d'intégration atteint dans la société flamande?

La réponse à ces questions sera soutenue par un ensemble de sources flamandes ${ }^{7}$, surtout des sources judiciaires de la ville de Bruges, distribuées dans le temps de la façon que nous pouvons voir sur le graphique 1 .

Graphique 1 - Registres documentaires sur la présence de marchands portugais en Flandre (1384-1449)

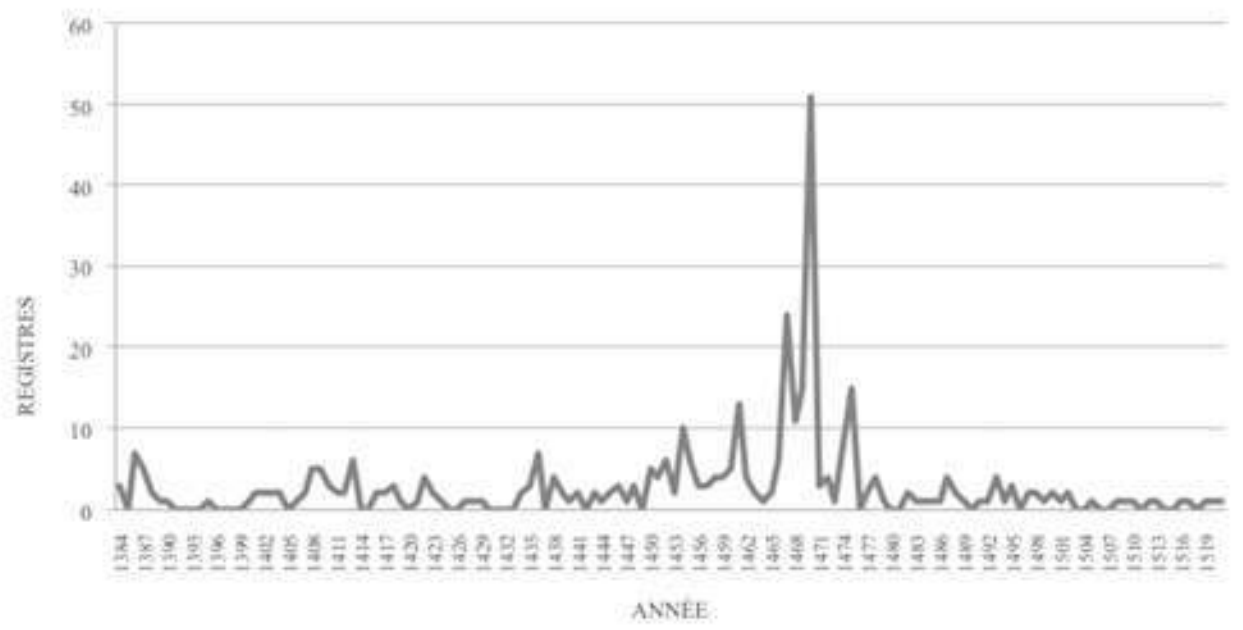

Le nombre de registres consignant des affaires impliquant des Portugais pour les 139 années étudiées n'est pas extraordinairement élevé. Sur un total de 369 registres, la moyenne est de seulement 2,67 par année. Il s'agit surtout de documents légaux, ce qui nécessite quelques précautions si nous voulons analyser l'intensité de la présence et de l'activité des marchands portugais en Flandre, parce que ces registres ne reflètent pas la dimension de ce qui fut leur commerce entre les deux territoires. Sur le graphique 1 on remarque deux périodes pendant lesquelles la moyenne est basse et constante : de 1384 jusqu'à 1449 avec 1,62 registre par an, et de 1477 jusqu'à 1521 avec 1,11 registre. La période centrale, entre 1450 et 1476, est caractérisée par 7,85 registres annuels, et il s'agit de la plus longue période de documentation ininterrompue, ce qui justifie cette division artificielle en trois temps sur lesquels nous nous appuierons pour exposer les faits ${ }^{8}$. La première période (1384-1449) correspond aux débuts des rapports commerciaux et diplomatiques entre le Portugal et la Flandre (les traités diplomatiques, l'hostilité entre les Nations et même la violence physique entre leurs membres) ; la deuxième (1450-1476), voit la croissance des échanges, des conflits commerciaux entre les marchands et des rapports avec les institutions judiciaires; dans la troisième (1477-1521) l'accent sera mis sur le rôle de la Nation portugaise à Bruges et ses relations avec la ville et le pouvoir ducal jusqu'au déplacement de la «Feitoria» de Flandre au Brabant (prêts consentis par la Nation à la ville de Bruges, utilisation de navires portugais comme vaisseaux de guerre et affirmation des droits individuels d'un marchand de Porto face à sa propre Nation).

\section{Diplomatie, commerce et guerre (1384-1449)}

5 La période A couvre 65 années et présente une moyenne assez modeste (1,62 registre par an), mais on voit déjà une présence constante sur une longue durée. Souvenons-nous que 
cette période contient quelques épisodes compliqués de l'histoire de l'Europe. Il est probable que la turbulence causée par la Guerre de Cent Ans soit à l'origine des nombreux traités de liberté de commerce entre la Flandre et le Portugal. Des 18 traités que j'ai pu étudier, seize ont été signés avant la fin du conflit, et 11 parmi ces 16 ont été rédigés entre 1384 et $1390^{\circ}$, c'est-à-dire, une période très difficile de l'histoire du Portugal, pendant laquelle le changement de dynastie finit par ranger les Portugais du côté de l'Angleterre (Graphique 2).

6 Des 139 registres conservés pour cette période, près de $10,4 \%$ correspondent à des cas de violence entre des Portugais et des membres d'autres Nationalités (Espagnols, Italiens et Flamands); les conflits entre les Portugais et les Espagnols représentent, à eux seuls, $5,6 \%$. Les occurrences les plus communes sont le pillage de navires et la violence physique, quelquefois avec l'emploi d'armes, et l'équilibre est clair entre les situations d'agresseur et de victime. Le nombre total de cas n'est pas très significatif, soit parce que le nombre de registres est réduit, soit parce que ceux-ci couvrent une période de 66 années.

$7 \quad$ Il est intéressant de constater que les conflits les plus fréquents sont ceux qui opposaient les Ibériques entre eux. Entre les Portugais et les Castillans, qui avaient lutté peu avant dans la terrible bataille d'Aljubarrota (14 août 1385), le ressentiment et l'amertume pourraient expliquer ces chiffres, puisque les trêves étaient précaires et furent plusieurs fois renouvelées jusqu'à la paix définitive en $1431^{10}$. Dans les documents où on voit les deux Nations engagées dans des scènes de violence ou de pillage, il est fait quelquefois mention du climat de guerre entre le Portugal et la Castille, ce qui sert tantôt de justification de la peine tantôt d'atténuation de celle-ci ${ }^{11}$. D'autres raisons ont sans doute poussé Antonio Pessagno, un Génois, à attaquer le Portugais Pedro Daniel ${ }^{12}$; ou Fernando Galdis, originaire de Galice, mais embarqué à bord d'un navire portugais, qui a blessé avec un couteau un marin portugais ${ }^{13}$.

Pendant ce temps-là, Philippe, duc de Bourgogne, qui en 1384 avait concédé aux Portugais la liberté de commerce, a renouvelé les traités à trois reprises en 1387, avec des articles spécifiques qui avaient pour but d'isoler les Anglais. Dans le premier de ces articles, daté du 15 janvier, il établit que la liberté de commerce n'inclut pas les affaires avec les Anglais ni avec leurs alliés ${ }^{14}$. Dans le deuxième, du 15 mai, il déclare que les assurances données aux marchands portugais ne seront valables que pour un $\mathrm{an}^{15}$. Dans le troisième, du 29 juillet, il explique que, en cas de supression des sauf-conduits, les marchands portugais devaient être prévenus et auraient trois mois pour préparer leur départ - ce qui permettait la continuité des affaires et la possibilité de quitter la Flandre sans pertes ${ }^{16}$. La stratégie de Philippe le Hardi empêchait un triangle commercial entre le Portugal, l'Angleterre et la Flandre, mais elle n'a pas pu faire obstacle à une relation bidirectionnelle anglo-portugaise. Avec la Gascogne affectée par la guerre et les Castillans luttant du côté de l'ennemi français, le vin portugais se présentait comme une vraie alternative $\mathrm{e}^{17}$ et le Portugal apparaissait comme une plateforme pour l'exportation de produits castillans vers l'Angleterre ${ }^{18}$.

9 La guerre de Cent Ans, en plus de toutes les conséquences économiques, politiques et sociales, a fonctionné comme élément de cristallisation du sentiment National, en mettant l'accent sur les caractères identitaires intrinsèques ${ }^{19}$. Coincés entre les Français et les Anglais, les ducs de Bourgogne, qui ont changé de camps plus d'une fois, ont été obligés de maintenir une politique d'équilibre, de neutralité, en même temps qu'ils poursuivaient une politique d'affirmation de leurs territoires ${ }^{20}$. Après le traité d'Arras, en 
1435, et une fois la position de Philippe le Bon envers l'Angleterre compromise ${ }^{21}$, les Flamands se voyaient exposés aux attaques anglaises ${ }^{22}$. Et les marchands portugais qui se dirigeaient vers la Flandre ont été eux aussi des cibles potentielles des corsaires et des pirates anglais ${ }^{23}$.

Graphique 2 - Types de litiges (1384-1449)

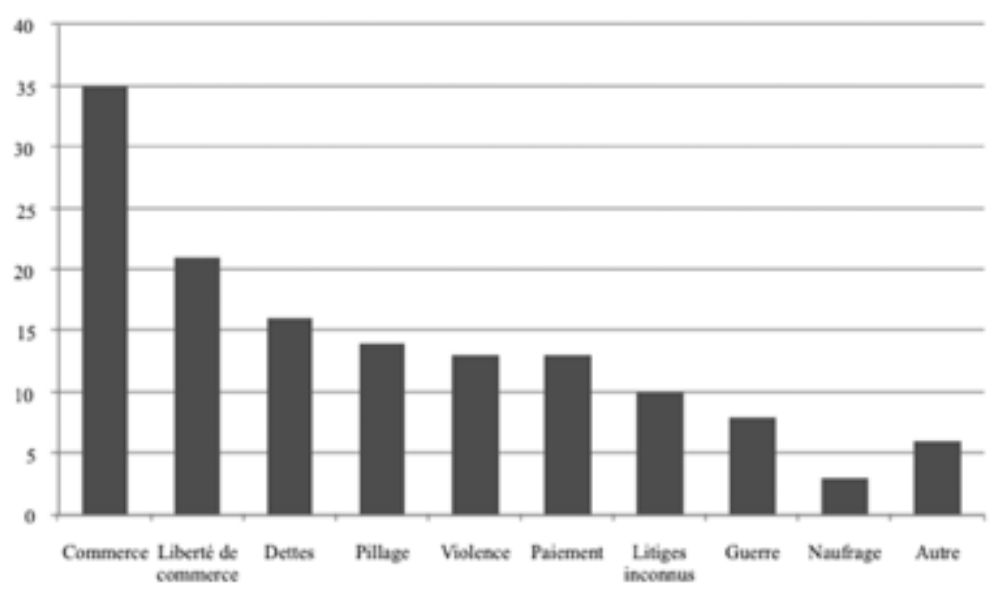

10 Le 17 novembre 1435, cinq bateaux du Portugal appartenant à Vasco Landim do Porto, Rodrigo Afonso, Gonçalo Afonso de Lisboa, João da Silva et Pedro Eanes , chargés avec du vin, des figues et des raisins secs, furent interceptés par des navires anglais et un de Bordeaux, sous le commandement du capitaine John Colville, qui les conduisit à Plymouth où les marchandises portugaises furent inventoriées. Après avoir examiné les registres d'achat et vente, les ravisseurs décidèrent de saisir la marchandise qui appartenait aux Flamands. Une partie du butin fut partagé entre le roi Henri VI et Humphrey, duc de Gloucester. Le reste revint aux Portugais et, suite à un ordre exprès du duc, leur fut effectivement restitué. Les marchands portugais reprirent leur route pour Bruges, en portant avec eux un message : la paix entre la Flandre et le roi de France signifierait une « guerre ouverte et de sang » avec l'Angleterre ${ }^{24}$.

11 Les marchands portugais allaient encore être victimes de l'hostilité entre Flamands et Anglais quelques années plus tard. En 1439, la ville de Bruges, au nom du duc de Bourgogne, envoya une lettre à Henri Beaufort demandant la restitution de 22 tonneaux d'huile et de deux sacs de farine, qui appartenaient à deux Portugais, Rui Sanches et Fernando Afonso ${ }^{25}$. Nous ne savons pas comment l'affaire s'est terminée.

La guerre est, en effet, un des éléments communs de presque tous les registres de cette période. Cela explique l'abondance des traités de commerce, dont la fonction légale était de protéger les affaires entre le Portugal et la Flandre. Les mentions de violence entre Portugais et Castillans ne faisaient que prolonger, au cœur de l'Europe, le conflit qui les opposait dans la péninsule Ibérique.

\section{Commerce et conflits (1450-1476)}

Une fois la paix définitivement établie entre Portugal et Castille, et la nouvelle dynastie d'Avis consolidée, le Portugal concentra davantage ses forces dans l'Atlantique ${ }^{26}$. Au-delà des produits traditionnels, la seconde moitié du $\mathrm{XV}^{\mathrm{e}}$ siècle est dominée par de nouvelles 
marchandises : le sucre ${ }^{27}$, l'ivoire ${ }^{28}$, les diamants ${ }^{29}$ et les épices ${ }^{30}$. Le sucre est sans doute la plus importante marchandise du commerce portugais en Flandre pendant cette période. Il est devenu un produit d'une énorme importance pour l'économie portugaise par le profit ${ }^{31}$ très élevé qu'il rapportait et par l'articulation de la production de l'île de Madeira avec les exigences des marchés euro-atlantiques et de la Manche, et a permis une transformation des modes de consommation et des pratiques alimentaires ${ }^{32}$.

Avec le temps, le nombre de produits vendus en Flandre a augmenté et les réseaux commerciaux et les affaires sont devenus plus complexes. On voyait de plus en plus de marchands portugais résidant en Flandre, dont les transactions concernaient, en plus des Flamands, des marchands italiens, espagnols, hanséates et anglais. À Bruges se réunissait un réseau paneuropéen des marchands, socialement organisés, profitant des institutions, des infrastructures et des mécanismes de négoce que la ville pouvait offrir: des auberges et des tavernes, des cambistes et des magasins, des églises et des bordels - et surtout le Grand Conseil pour les questions judiciaires ${ }^{33}$.

La période B (1450-1476) couvre 26 années et représente le maximum de l'activité des marchands portugais en Flandre (7,85 registres annuels), du moins d'après les documents étudiés à ce jour. Si on analyse une fois de plus la distribution des registres par sujet (graphique 3), on observe une croissance significative des disputes provoquées par des dettes (121 registres), ainsi qu'un nombre croissant de litiges dont le motif n'est pas spécifié (31 registres pour lesquels il est difficile d'établir une typologie parce que les documents indiquent seulement l'ouverture d'un procès, sans expliquer de quoi il s'agit). On constate aussi une légère croissance du nombre de cas de violence (13\%). Dans le même temps que les traités de liberté de commerce deviennent résiduels (moins de $1 \%$ ).

Graphique 3 - Types de litiges (1450-1476)

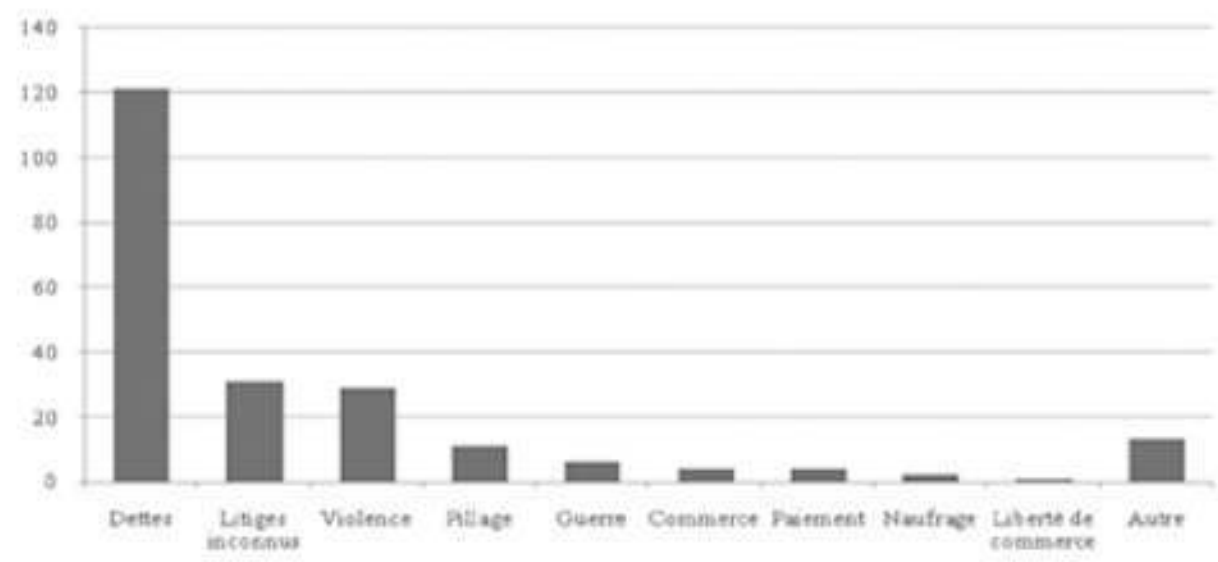

Entre 1450 et 1476 on trouve dans la documentation 110 noms de Portugais, au regard de 49 Flamands, 20 Italiens, 11 Espagnols et 3 Hanséates $^{34}$. Ces chiffres sont significatifs si nous prenons en compte la période envisagée - seulement 26 années - et le fait qu'un grand nombre de ces marchands portugais résident effectivement à Bruges; il y en a qui y habitent durant plus d'une décennie.

D'autre part, par leur nature même, ces documents témoignent des conflits, et non pas des affaires conclues avec succès et sans problèmes. Ces cas de dettes se rapportent à des 
affaires dont la résolution n'a été possible qu'après la médiation des magistrats de Bruges ou d'arbitres élus ad hoc. Cette dernière situation se vérifie souvent quand les litiges opposent des marchands de différentes nationalités: on crée alors une commission d'arbitrage de quatre membres ${ }^{35}$. Dans le cas du conflit opposant Rombout de Wachtere à Martim Lem, tous deux citoyens de Bruges ${ }^{36}$, au sujet de bijoux d'une grande valeur ${ }^{37}$, une de ces commissions de quatre personnes a été nommée et l'un d'eux était un Portugais ${ }^{38}$. Le cas allait traîner plusieurs mois et la commission changea de structure par la suite, mais de tels cas nous révèlent les mécanismes de protection et d'appel à la justice, ainsi que le haut niveau d'intégration des étrangers dans la société flamande. On voit parfaitement cette intégration dans les registres dans lesquels les marchands portugais sont référencés comme des citoyens de Bruges ${ }^{39}$. La citoyennetée ${ }^{40}$, en plus d'assurer à ceux qui la recevaient les mêmes droits que n'importe quel citoyen de Bruges, rendait possible un plus grand éloignement de la Nation du Portugal ${ }^{41}$. Quand une dispute entre des marchands portugais avait lieu et que l'un des intéressés avait la citoyenneté de Bruges, la Nation du Portugal cessait d'avoir juridiction pour résoudre le conflit. Un exemple : la Nation avait entre ses mains la décision d'un litige entre Gonçalo Afonso et Rui Machado, mais ce dernier avait le statut de citoyen. L'Assemblée des Magistrats de Bruges informa les consuls portugais - Luís Vicente et Gil Eanes - qu'ils ne pouvaient pas juger des cas touchant des citoyens de la ville ou d'une autre nationalité, parce que cela constituait une entorse aux droits et aux principes établis.

Mais beaucoup de cas étaient résolus au sein de la Nation du Portugal, qui protégeait et aidait les marchands en cas de nécessité. Naturellement, les marchands n'étaient pas toujours d'accord avec les décisions de la justice de leur Nation. Au cours de cette période, nous trouvons cinq registres d'appel des sentences prises par les consuls portugais ${ }^{42}$. Les mécontents recouraient alors à la justice de la ville. En dernière instance, si le privilegium fori était invoqué, cet appel était jugé par le Grand Conseil des Pays-Bas, à Malines ${ }^{43}$.

19 L'année 1470 voit culminer le nombre de registres sur la présence de marchands portugais en Flandre. Elle commence avec une altercation entre deux Portugais, à l'Écluse, au début de janvier ${ }^{44}$, pour finir, le 31 décembre, avec un accord entre le marchand portugais Álvaro Dinis et l'Anglais John Raes ${ }^{45}$. Mais la Flandre connaît alors une période de transformations politiques sous le gouvernement de Charles le Téméraire. Pendant dix ans, comme duc de Bourgogne (1467-1477), Charles s'est montré impulsif et inflexible, et a plongé ses territoires dans une guerre continue avec la France ${ }^{46}$. Au mois de mai de 1470 nous savons qu'au moins seize navires portugais étaient en Flandre, et qu'ils ont été affrétés et intégrés dans la flotte du duc Charles, pour la période comprise entre le 9 mai et le 7 juin $^{47}$. Ils n'ont pas pu échapper à une guerre qui n'était pas la leur, mais ils ont pu sauvegarder leurs intérêts en obtenant des contreparties financières. Le duc a payé la location de chaque bateau, et une solde à chaque membre de l'équipage. La cinquième partie des registres de cette année est en rapport avec cette affaire. Si le duc Charles n'avait pas réquisitionné des navires étrangers pour sa flotte, nous ne saurions pas qu'au mois de mai de 1470 plus d'une dizaine de marchands portugais se trouvait en Flandre ${ }^{48}$.

\section{L'abandon de Bruges (1477-1521)}

20 À la fin des années 1480 on assiste à l'effondrement de la ville de Bruges comme grand centre de commerce international. Cela explique la diminution soudaine du nombre de 
registres pour la période $C$ à un peu plus d'un registre annuel (Graphiques 1 et 4). Les conflits entre le duché de Bourgogne et la France, les altérations du lit du Zwin, l'avènement d'Anvers et les problèmes financiers de Bruges contribuèrent à précipiter la ville dans une crise. Pour cette période finale, trois cas retiendront notre attention.

Le premier se passa en décembre de 1487. L'activité de changeur à Bruges, comptait sans doute parmi les plus importantes et les plus rentables de ce temps. Mais cette activité ne s'est pas bien terminée pour Willem Roelens, qui fit faillite et dut faire face à ses créanciers hanséates, espagnols et portugais. Il est possible que la Nation portugaise ait été laissée de côté lors d'un premier accord ; elle a donc exigé du Grand Conseil de Bruges un traitement semblable à celui reçu par les Hanséates et les Espagnols - c'est-à-dire, le payement échelonné de la dette par la ville. Dans ces situations, la ville de Bruges devait assumer le privilège accordé aux marchands étrangers selon lequel elle restituerait la somme totale due ${ }^{49}$. Le consul portugais, Lopo Pires, obtint que les 213,13 livres soient intégralement payés en trois ans, en deux versements, un à la Saint Jean, l'autre à Noël ${ }^{50}$. Les intérêts de la Nation portugaise étaient assurés, ainsi que l'égalité de traitement vis-àvis des autres Nations.

Les dettes de la ville de Bruges envers les marchands portugais ne finirent pas là. L'année suivante, avec l'insurrection qui conduisit à la séquestration de l'empereur Maximilien, la ville emprunta de l'argent à des marchands étrangers ${ }^{51}$. L'épisode allait se révéler tragique pour Bruges, dont l'importance comme centre d'échanges avait été réduite à des proportions insignifiantes ${ }^{52}$.Quelques années plus tard, puisque la ville s'avouait incapable de payer ses dettes (20 625 florins d'or et 230 livres), la Nation portugaise décida de saisir des marchandises transportées par des citoyens de Bruges. Après la paix de Montils-lès-Tours, signée le $1^{\text {er }}$ octobre 1489, l'empereur Maximilien força la ville de Bruges à rembourser les créanciers pour toutes les dettes contractées, qui se montaient à 150000 écus d'or. Assez endettée, elle a essayé de passer des accords avec tous les créanciers. Afonso Martins, facteur du Portugal, et les autorités de Bruges, décidèrent que la dette serait payée en quatre prestations annuelles de 5156,25 florins. La première prestation devait être payée le $1^{\mathrm{er}}$ novembre 1492 , mais elle ne le fut pas. Dans un document de 1493 présenté devant le Grand Conseil de Malines, le facteur du Portugal, Afonso Martins, confirme qu'il a ordonné la saisie des marchandises transportées par deux citoyens de Bruges, Jacob Despars et Hendrick Nieuwland. Cette saisie a été validée par les échevins du Grand Conseil, parce que, en tant que citoyens de cette ville, ils ont été forcés, in solidum, d'assumer les dettes de la communautés3. 


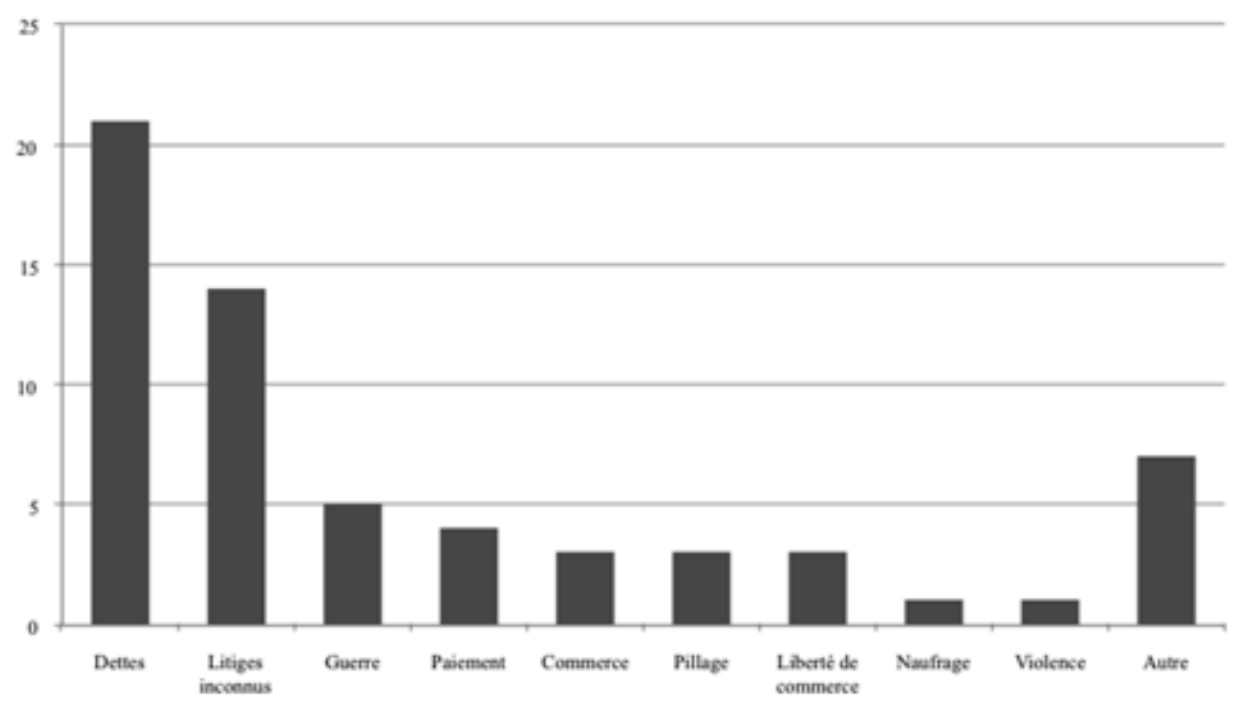

Dans les années suivantes, les marchands portugais ont abandonné Bruges pour Anvers ${ }^{54}$. En 1516, Lopo de Calvos, de Porto, était le seul marchand portugais encore présent à Bruges. Comme il ne payait pas les droits dus à la Nation portugaise ${ }^{55}$, celle-ci porta plainte devant le Grand Conseil des Pays-Bas. Mais le marchand produisit une assez bonne défense, en invoquant les privilèges concédés par le duc Philippe de Bourgogne : celui qui ne paie pas les droits à sa Nation ne bénéficie pas des privilèges. En plus, la Nation portugaise avait refusé de l'aider quand il avait perdu un navire chargé de marchandises. C'est alors que, révolté, il décida de ne plus payer les droits ${ }^{56}$. Le grand Conseil décida en faveur du marchand de Porto. Si dans d'autres cas la Nation portugaise avait fonctionné comme institution de protection et intégration des marchands, les nombreux cas d'appel contre des sentences de cette Nation et l'épisode de Lopo de Calvos que nous venons de résumer montrent que, pour les Portugais, le danger ne venait pas toujours des étrangers.

\section{Dernières considérations}

Les documents disponibles s'insèrent dans une catégorie spécifique : ce sont surtout des sources produites par la chancellerie du duché de Bourgogne et par les magistrats du Conseil de Bruges. Les circonstances d'élaboration sont particulières: régulation du commerce ou résolution de conflits. Ces circonstances peuvent fausser notre perspective du commerce médiéval, suggérant qu'il alimentait quantité d'actions en justice, que les marchands étaient majoritairement des escrocs et les marins des brigands. Ce qui est tout à fait faux. Quoique nous ayons mentionné, au long de ce travail, des conflits et des problèmes, le rapport le plus normal était un rapport de confiance. Confiance aux agents, aux nouveaux mécanismes de la finance, aux marchands des autres Nations, aux contacts locaux et aux institutions ${ }^{57}$.

Confiance dans le commerce international, mais conscience des dangers, comme un naufrage ou une attaque en haute mer. Ces dangers pouvaient être raisonnablement couverts par les assurances qui protégeaient le risque de l'investissement effectué, mais aussi à travers les mécanismes de la justice - recours contre les dettes, les pillages, la 
violence et la guerre. En mettant en contexte les cas analysés, nous voyons bien que les marchands portugais connaissaient les lois et protestaient quand ils pensaient que le droit n'était pas appliqué d'une façon équitable. Parfois ils ont vu leur droit reconnu, d'autres fois ils ont perdu les litiges qui les opposaient à des marchands d'autres nationalités.

La Nation du Portugal - organisation légalement encadrée et autorisée grâce aux privilèges du comte Philippe, à la fin du XIV ${ }^{e}$ siècle - a réuni les marchands et fait valoir sa juridiction pour les questions internes. Mais le plus important c'est de savoir qu'il y avait des procédures légales qui permettaient les appels, puisque la justice n'était pas toujours juste: appel donc de la Nation devant le conseil des magistrats de Bruges; et de celui-ci devant le Grand Conseil des Pays-Bas.

Justice, donc, mais plus encore, intégration à travers la Nation et les aubergistes, qui souvent connaissaient bien la langue et les habitudes de leurs hôtes. Jakop van Le Fine, par exemple, s'est spécialisé dans l'hébergement de marchands portugais et espagnols ${ }^{58}$. Guillaume Baerber qui, en janvier de 1455 accueillait dans son auberge au moins cinq marchands portugais ${ }^{59}$, offraient des chambres, de la nourriture, un espace pour ranger leurs marchandises et même un système de crédit dont l'importance dépassait les limites de l'auberge et même de la ville ${ }^{60}$.

Cela dit, les marchands portugais se sentaient-ils des étrangers en Flandre, pour reprendre la question initiale? Probablement oui. Sans doute, ils n'ont jamais cessé de s'étonner de la langue et des usages flamands. Probablement ils ne se sont jamais adaptés au climat et à la nourriture. Mais ils sont peut-être devenus des étrangers assez bien intégrés, parce que les institutions le permettaient, de même que les structures et le cosmopolitisme de Bruges, ville marquée par la pénétration des valeurs monétaires et commerciaux dans tous les domaines de la vie sociale ${ }^{61}$. Cela explique que quelques marchands portugais aient choisi Bruges comme centre pour leurs affaires, en y habitant pendant plus de dix ans. Et aussi comme " centre affectif», puisque quelques-uns d'entre eux y fondèrent une famille en épousant des Flamandes et étaient capables de parler l'idiome local ${ }^{62}$.

Confrontés à divers types d'hostilités, les marchands portugais ont recouru à la justice pour défendre leurs intérêts et se protéger d'éventuelles inégalités. Mais il est essentiel de souligner l'énorme importance de la liberté individuelle et du comportement politique de la Flandre ${ }^{63}$, qui vivait du commerce et qui créa les meilleures conditions pour sa concrétisation $^{64}$. Nous pouvons toujours penser, bien sûr, que les Portugais étaient vus comme des amis et non pas comme des étrangers ${ }^{65}$, en confirmant l'idée de Fernand Braudel selon laquelle les mouvements des marchands ont représenté une révolution qui est passée de l'économie à la conduite sociale ${ }^{66}$.

\section{NOTES DE FIN}

1. Cette étude a été développée au cours de mon séjour à l'Université de Groningen (2007-2008), grâce à une bourse Marie Curie Fellowship (European Doctorate in the Social 
History of Europe and the Mediterranean). Je remercie les professeurs Luís Miguel Duarte (université de Porto), Hilario Casado Alonso (université de Valladolid) et Michel Bochaca (université de La Rochelle).

2. BLockMANS, Wim, PREVENIER, Walter, The Promised Lands, The Low Countries Under Burgundian Rule, 1369-1530, Philadelphia, University of Pennsylvania Press, 1999, p. 5. 3. SICKING, Louis, « Le paradoxe de l'accès : le rôle des avant-ports dans les Pays-Bas à la fin du Moyen Âge et au début de l'époque moderne (approche comparative générale) »; BOCHACA, Michel, SARRAZIN, Jean-Luc, Ports et littoraux de l'Europe atlantique, Rennes, PUR, 2007, p. 227-255. Voir aussi : MURRAY, James M., Bruges, Cradle of Capitalism. 1280-1390, Cambridge, CUP, 2005, p. 22 sqq.

4. BLOCKMANS, Wim, PREVENIER, Walter, The Promised Lands..., op. cit.,p. 5, 6, 47. Le concept a été proposé par l'historien Philippe de Commynes.

5. BLONDÉ, Bruno, GELDERBLOM, Oscar, STABEL, Peter, « Foreign Merchant communities in Bruges, Antwerp and Amsterdam, c. 1350-1650 ", CALABI, Donatella, CHRISTENSEN, Stephen (dir.) - Cultural Exchange in Early Modern Europe. Cities and Cultural Exchange in Europe 1400-1700, vol. II, Cambridge, CUP, 2007, p. 154. Voir aussi : GODINHO, Vitorino Magalhães, A Expansão Quatrocentista Portuguesa, Lisboa, D. Quixote, 2008, p. 44.

6. Les rapports entre le Portugal et la Flandre remontent au XII ${ }^{\mathrm{e}}$ siècle, quand Philippe d'Alsace (1143-1191), comte de Flandre, s'est marié, en 1183, à la princesse Teresa, fille d'Afonso Henriques, premier roi du Portugal. Plus tard, en 1430, une autre princesse, Isabelle, fille de João I, se rendit en Flandre pour épouser Philippe le Bon, duc de Bourgogne (1396-1467). Les pèlerinages et les Croisades furent un autre facteur de rapprochement des deux territoires. Si les chevaliers et les marchands du Nord (Hanséates, Flamands, Anglais) sont passés par le Portugal pour aller combattre les Sarrasins dans la péninsule Ibérique ou en Terre Sainte, curieusement, le processus s'est inversé au XIv ${ }^{\mathrm{e}}$ siècle. Plusieurs combattants des Pays-Bas, d'Angleterre, de France, d'Écosse, de Florence et du Portugal ont rejoint les rangs des Chevaliers Teutoniques en lutte contre les Lituaniens de l'Est de la Prusse. Sur les Croisades et les pèlerinages en Ibérie : MARQUeS, A. H. de Oliveira, Hansa e Portugal na Idade Média, Lisboa, Presença, 1993, p. 28-41 ; sur les Portugais contre les Lituaniens : MURRAY, James M., Bruges..., op. cit., p. 245-246; pour un résumé des rapports économiques entre le Portugal et la Flandre : PAVIOT, Jacques, «Les relations économiques entre le Portugal et la Flandre au $\mathrm{XV}^{\mathrm{e}}$ siècle ", Congresso Internacional Bartolomeu Dias e a sua Época, Porto, Universidade do Porto, vol. III, 1989, p. 531-539.

7. FREIRE, Anselmo Braamcamp (ed.), « Maria Brandoa, a do Crisfal », Archivo Historico Portuguez (AHP), Vol. VII, 1909. UNGER, W. S., Bronnen tot de geschiedenis van Middelburg in den landsheerlijken tijd (BvM), Den Haag, 1926, 1931. NIERMEYER, J. F., Bronnen voor de economische geschiedenis van het Beneden-Maasgebied, Den Haag, 1968 (désormais BvB). MARQUES, João Silva, Descobrimentos Portugueses, Lisboa, INIC, 1988 (désormais DP). ANSWAARDEN, Robert van (ed.), Les Portugais devant le Grand Conseil des Pays-Bas (1460-1580, Paris, Centre Culturel Calouste-Gulbenkian, 1991(désormais PGC). PAVIoT, Jacques (ed.), Portugal et Bourgogne au Xve siècle (PB), Paris, Centre Culturel Calouste Gulbenkian, 1995. PAVIOT, Jacques, « Les Portugais à Bruges au XV siècle ", Arquivos do Centro Cultural Calouste Gulbenkian, vol. 38, Lisboa, Gulbenkian, 1999, p. 1-122 (désormais LPB).

8. La division reste artificielle et représente un changement notable dans l'activité commerciale des Portugais en Flandre. Alors que nous nous trouvons devant une suite ininterrompue de registres, les années marquées par d'importants évènements politiques 
et militaires au Portugal, nous n'avons aucune trace de marchands portugais en Flandre. Tel est le cas de 1449, année de la bataille d'Alfarrobeira, au Portugal, entre le roi Afonso v et l'infant Pedro, son oncle. De même en 1476, où les Portugais et les Castillans s'opposent à la bataille de Toro. Cette absence se vérifie en 1385 (bataille d'Aljubarrota) ; en 1415 (prise de Ceuta, au Maroc) ; 1437 (échec de la prise de Tanger). Il y a cependant des exceptions à cette règle : en 1458 (prise d'Alcácer Ceguer), 4 registres nous sont parvenus ; 1471 (prise d'Arzila), 3 registres, en fort contraste avec l'année antérieure, 1470 (51 registres). À partir de ces chiffres, pourrait-on penser que les bateaux des marchands - et, peut-être même, les hommes - ont participé à l'effort de guerre, ce qui les aurait détournés de l'activité marchande?

9. BvM, vol. 3, doc. 3, 59, 99; BvB, vol. 1, doc. 620; PB, doc. 1, 2, 3, 4, 5, 6, 9.

10. SouSA, Armindo de, «Realizações », MATTOso, José, História de Portugal, A Monarquia Feudal (1096-1480), Lisboa, Estampa, 1993, p. 499.

11. $\mathrm{PB}$, doc. $24,28$.

12. $\mathrm{PB}$, doc. 17 .

13. $\mathrm{PB}$, doc. 10

14. $\mathrm{PB}$, doc. 4.

15. $\mathrm{PB}$, doc. 5 .

16. $\mathrm{PB}$, doc. 6.

17. SPUFFORD, Peter, Power and Profit, The merchant in medieval Europe, London, Thames \& Hudson, 2002, p. 294.

18. CHILDS, Wendy, Anglo-Castilian Trade in the Later Middle Ages, Manchester, Manchester University Press, 1978, p. 42. La vraie dimension du Portugal comme plateforme d'exportation des produits de Castille est encore à étudier, mais il est certain que le rapprochement entre l'Angleterre et la Castille a rétabli la normalité dans les routes maritimes.

19. MOAL, Laurence, L'Étranger en Bretagne au Moyen Âge, Rennes, PUR, 2008, p. 17-19.

20. BLocKmAns, Wim, PREVEnIER, Walter, The Promised Lands..., op. cit., p. 75-86. «Philips's marriage to Isabella of Portugal in 1430 had signaled another dimension of his new policy : neutrality with respect to France and England and the ambition to demonstrate to the rest of Europe that the Burgundian state itself had achieved the status of a great power $»$.

21. MINOIS, Georges, La Guerre de Cent Ans, Paris, Perrin, 2008, p. 368-372. Voir aussi : BLOCKMANS, Wim; PREVENIER, Walter, The Promised Lands..., op. cit., p. 81 ; MACKAY, Angus, DitchBuRn, David (eds.), Atlas de Europa Medieval, $2^{\mathrm{e}}$ ed., Madrid, Cátedra, 2006, p. 179-184. 22. CURVEILLER, Stéphane, "Complémentarités et rivalités des ports maritimes en Flandre occidentale à la fin du Moyen Âge ", Ports Maritimes et Ports Fluviaux au Moyen Âge, Paris, Publications de la Sorbonne, p. 251. Susan Rose signale une attaque à Calais à laquelle ont participé des navires portugais : ROSE, Susan, Medieval Naval Warfare, 1000-1500, London, Routledge, 2002, p. 81-99. À propos de Calais, voir aussi : CURVEILLER, Stéphane, «Calais and Dunkirk : Two Sentinels, Two Geo-Strategic States », International Journal of Maritime History, XIX, No. 2, December 2007, p. 287-297.

23. Sur la Flandre et la guerre de Cent Ans : PISTONO, Stephen P., Flanders and the Hundred Years War : the Quest for the Trêve Marchande, Institute of Historical Research, vol. 49, n. 120, 1976, pp. 185-197. La question de la violence littorale pendant la guerre de Cent Ans, qui affecta les routes commerciales, est aussi mentionnée dans : ARÍZAGA BOLUMBURU, Beatriz, BOCHACA, Michel, TRANCHANT, Mathias, « La violence en mer et dans les ports du golfe de 
Gascogne à la fin du Moyen Âge : bilan et perspectives de recherche ", AUGERON, Mickaël et TRANCHANT, Mathias (dir.), La Violence et la Mer dans l'espace atlantique (XII ${ }^{e}-X_{X}{ }^{e}$ siècle), Rennes, PUR, 2004, p. 28-32 ; aux pages 37 et 38 il y a deux registres de Portugais engagés dans des conflits près de La Rochelle.

24. $\mathrm{PB}$, doc. 184, 185.

25. $\mathrm{LPB}$, doc. 3.

26. Sur l'expansion océanique portugaise : BETHENCOURT, Francisco, CURTO, Diogo Ramada (dir.), Portugueses Oceanic Expansion, 1400-1800, Cambridge, CUP, 2007 ; DAVIS, Ralph, The Rise of the Atlantic Economies, London, Fakenham and Reading, 1973, p. 1-14 ; FINDLAY, Ronald, O'ROURKe, Kevin, Power and Plenty, Trade, War, and the World Economy in the Second Millenium, Princeton, Princeton University Press, p. 145-166 ; GoDINHO, Vitorino Magalhães, $A$ Expansão Quatrocentista..., op. cit.

27. $\mathrm{LPB}$, doc. 83. Il y a 11 registres sur le sucre en tant que produit du commerce; le premier date de 1468 ; Bruges, et plus tard Anvers, ont absorbé une grande partie du sucre provenant de la « terre aux nombreuses cannes ».

28. $\mathrm{LPB}$, doc. 49. PGC, doc.4.

29. $\mathrm{LPB}$, doc. 20. Un autre registre mentionne des diamants, mais il s'agit d'un achat à des Flamands : PB, doc. 33.

30. À propos des épices : FREEDMAN, Paul, Out of the East, Spices and the Medieval Imagination, Yale, Yale University Press, 2008, p. 193-205.

31. D'après des chiffres du Livro do almoxarifado dos açuquares das partes do Funchal, en 1494 la production a atteint 80451 arrobes. Puisque la valeur moyenne de l'arrobe, en 1496, variait entre 350 et 600 " reais », il est facile de comprendre que l'affaire du sucre est devenue très lucrative, attirant des marchands étrangers. L'île de Madère a vu arriver surtout des Italiens et des Flamands, qui ont bénéficié pendant quelque temps de la liberté de commerce. Mais au cours de la deuxième moitié $d u \mathrm{XV}^{\mathrm{e}}$ siècle, la spéculation finit par provoquer des variations dans les prix, portant préjudice aux producteurs et à quelques marchands. Cela provoqua une intense activité législative, soit de la Couronne soit de la ville du Funchal. Voir : RAU, Virgínia, MACEDO, Jorge de, 0 açúcar da Madeira nos finais do século XV, Funchal, Junta Geral do Distrito Autónomo do Funchal, 1962, p. 13 ; VIEIRA, Alberto, «A Madeira e o mercado do açúcar. Séculos XV-XVI », História do açúcar : rotas e mercados. Funchal, Centro de Estudos de História do Atlântico, 2002, p. 58, 59, 72, 77; DP, vol. III, doc. 45.

32. Le Portugal produisait du sucre depuis 1404. On a essayé de le cultiver dans l'Algarve (Quarteira) et plus tard à Coimbra. Mais on n'arriva à une production en quantité et qualité satisfaisantes que lorsqu'on le planta à Madeira. Très rapidement, le produit commença à être présent avec fréquence dans les marchés internationaux : Bristol, en 1466, et Bruges, deux années plus tard. RAU, Virgínia, MACEDO, Jorge de, 0 açúcar da Madeira..., op. cit., p.11.

33. MURRAY, James M., Bruges..., op. cit., p. 219. Un réseau social de marchands et un développement institutionnel assurèrent l'efficacité des services et des affaires et conduisirent à l'affirmation de Bruges : « And it was the number and complexity of these institutions and their contacts that made Bruges the first "network" city in European economic history ». À la p. 190 : "The deus ex machina of such a trading system was an increasing force of attraction to the Bruges market that made it imperative for a merchant to have a presence there.

$\gg$ 
34. Cela se passe tout au long de 213 registres ; plusieurs cas se prolongent dans le temps et ont d'autres registres avec des suites.

35. PAVIOT, Jacques, Bruges, 1300-1500, Paris, Autrement, 2002, p. 48.

36. Il y a dans les sources deux marchands portugais qui sont présentés simultanément comme des citoyens de Bruges. Mais le cas de Martim do Lem est différent, parce que, quoiqu'il soit référencé une fois comme portugais, il est originaire du Brabant. Un long séjour à Lisbonne, où il a eu sept fils qui ont hérité de ses affaires, explique sans doute son « changement » de nationalité. Voir : LEME, Margarida, Os Lemes - um percurso familiar de Bruges a Malaca, Sapiens, História, Património e Arqueologia. nº 0, décembre 2008, p. 51-83. BLONDÉ, Bruno, GELDERBLOM, Oscar, STABEL, Peter, « Foreign Merchant communities in Bruges, Antwerp and Amsterdam, c. 1350-1650 », op. cit., p. 156, 157. « Most merchants stayed in the city for a short period of time, but a good number of them lived there for longer periods, or perhaps even resided for a lifetime. [...] Some Genoese and Castilians bought citizenship and integrated themselves into local elites through marriage. Their integration gave them a specific role as intermediaries between local society and other interNational merchants. " 37. LPB, doc. $53,56,57,60,62,63,66,68,69,70,77$. Le procès dura plus d'un an jusqu'à sa conclusion. Le document avec la sentence n'est pas complet et les pièces antérieures donnent peu d'informations sur le conflit. Le litige porte sur 1310 écus d'or à cause de bijoux qui devaient être vendus à Lisbonne. Les contours de l'histoire ne sont pas clairs. Wachetere accusa Lem d'avoir fait de mauvaises affaires à Lisbonne et à Séville.

38. Pieter Metteneye, Lievin vander Mersch, Zeghere Parmentier et João Esteves. Le portugais João Esteves est présent dans la documentation flamande entre 1455 et 1470 .

39. LPB, doc. 6 .

40. Bruges serait une des villes avec le plus d'étrangers au Moyen Âge, et entre 1281 et 1408 près de 20.000 personnes ont acquis le droit de citoyenneté : MURRAY, James M., Bruges..., op. cit.,p. 304.

41. La Nation du Portugal bénéficiait depuis la fin du XIV ${ }^{\mathrm{e}}$ siècle d'un statut juridique spécifique : ANSWAARDEN, Robert van, Les Portugais devant le Grand Conseil..., op. cit., p. 38. Pour d'autres informations sur la Nation du Portugal, ses fonctions et son statut, voir le même auteur : p. 41-47 ; 53-70. Van Houtte affirme qu'il y avait beaucoup de Nations ibériques à Bruges : HOUTTE, J. A. Van, An Economic History of the Low Countries, 800-1800, London, Weidenfeld and Nicolson, 1977, p. 101.

42. $\mathrm{LPB}$, doc. $71,75,81,85,86$.

43. PGC, doc. 1. Voir les commentaires de Robert van ANSWAARDEN, p. 106.

44. PB, doc. 65.

45. LPB, doc. 132 .

46. BLOCKMANS, Wim, PREVENIER, Walter, The Promised Lands..., op. cit.,p. 174-205.

47. PB, doc. 420 .

48. Les documents sur cet épisode fournissent d'autres données intéressantes sur le tonnage des bateaux, l'équipage et la paye reçue.

49. En cas de faillite, la responsabilité d'un changeur retombait sur la ville de Bruges tout entière, laquelle, déjà au XIV ${ }^{\mathrm{e}}$ siècle, avait été forcée d'acquitter les dettes de trois changeurs : Collard de Marke, Boiden Weghebedde et une certaine Nanne, veuve de Diederic Urbaens. Au milieu du XIV ${ }^{\mathrm{e}}$ siècle, la ville a pris des mesures pour résoudre le problème de la faillite des changeurs : «By the middle of the fourteenth century, supervision of bankruptcies had been entrusted to the "burgomaster of the Course", the chief of the second bank of Bruges aldermen. He typically presided over the seizure of the records and properties of the 
failed money changer. He then proceeded to an audit of the assets and debts owed on the basis of records and testimony, and supervised the distribution of a proportionate share of the recovered funds to creditors. In so doing he was obliged to observe privileges that granted some foreign merchants a right to full restitution... ", dans MURRAY, James, Bruges..., op. cit., p. 157.

50. LPB, doc. 154.

51. La mort de Charles le Téméraire, en 1477, plongea ses domaines au cœur d'une dispute entre les Habsbourg et la France. Les mesures prises par la jeune duchesse Marie, qui a restitué des libertés et des garanties, n'ont pas permis à Bruges de récupérer totalement l'importance de jadis. Voir : BLOCKMANS, Wim, PREVENIER, Walter, The Promised Lands..., op. cit.,p. 196-205 et PAVIOT, Jacques, Bruges..., op. cit., p. 26-27.

52. PAVIOT, Jacques, Bruges..., op. cit., p. 27-28. BLOCKMANS, Wim, PREVENIER, Walter, The Promised Lands..., op. cit.,p. 199-203.

53. PGC, doc. 11. Dans ce document est mentionné Jacob Despars, frère de Wouter Despars, qui négociait du sucre et d'autres produits au Portugal. Voir : SPUFFORD, Peter, Power and Profit..., op. cit., p. 309.

54. AHP, doc. XIX. En 1493, la ville de Bruges a attribué des privilèges aux marchands portugais, mais Anvers est très vite devenue le grand entrepôt commercial de la région avec des garanties concédées en 1488 par l'empereur Maximilien. Voir aussi : AHP, doc. XVIII. 55. Depuis 1293, pendant le règne de Dinis (1279-1325), les marchands portugais étaient forcés de donner un pourcentage de leurs profits pour une bourse de marchands. Cette arca communis était une sorte d'assurance. Voir : ANSWAARDEN, Robert van, Les Portugais devant le Grand Conseil..., op. cit., p. 53-56.

56. PGC, doc. 24.

57. Wendy R. Childs (Anglo-Castilian trade..., op. cit., p. 189), affirme : « A study of the legal documents always leaves the impression that trade was just about to founder under widespread dishonesty, cheating, violence, stupidity and jealousy, but the cases where things went wrong were only a small fraction of the total number of deals and on the whole merchants could trust their agents, servants and contacts ». Bien qu'étudiant une période différente et le rapport entre la loi et l'économie, Erin Ann O'Hara (dans o'HARA, Erin Ann, « Trustworthiness and Contract », ZAK, Paul J. (ed.), Moral Markets, The Critical Role of Values in the Economy, Princeton, Princeton University Press, 2008, p. 173) écrit que « Trust and trustworthiness work hand-in-hand [...] and the two together can contribute to economic efficiency ". Cet aspect a été également souligné par Peter Spufford (Money and its use in medieval Europe, Cambridge, CUP, 1988, p. 254.), qui montre l'importance de la confiance dans le développement du crédit et du commerce.

58. MURRAY, James M., Bruges..., op. cit., p. 241-242.

59. LPB, doc. 25, 93. Dans d'autres documents (17, 24, 27, 28, 50, 51, 58, 82, 92, 107, 129), nous voyons Filip van Aertrijcke, citoyen de Bruges, héberger ou servir de caution à des marchands portugais, ce qui confirme peut-être cette spécialisation.

60. MURRAY, James M., Bruges..., op. cit., p. 192.

61. KAYE, Joel, Economy and Nature in the Fourteenth Century, Money, Market Exchange, and the Emergence of Scientific Thought, Cambridge, CUP, 1998, p. 15-36. Dans son premier chapitre, J. Kaye conteste la conscience monétaire des sociétés aux XIII ${ }^{\mathrm{e}}$ et XIV ${ }^{\mathrm{e}}$ siècles.

62. LPB, doc. 23, 97.

63. Avner Greif analyse l'importance de caractéristiques non technologiques - les institutions - comme fondamentales pour le développement économique au Moyen Âge, parce qu'elles articulent les lois avec leur application, en protégeant les droits 
individuels, en combattant la corruption et assurant la confiance. Il compare cette situation avec les sociétés qui ont réussi à développer plus efficacement ces caractéristiques, avec l'objectif de démontrer le succès économique des régions les plus développées du point de vue plus institutionnel. GREIF, Avner, Institutions and the Path to the Modern Economy, Lessons from Medieval Trade, Cambridge, CUP, 2006, p. 1-7, 58-65.

64. EPSTEIN, S. R., Freedom and Growth, The rise of states and markets in Europe, 1300-1750, London, Routledge, 2000, p. 12.

65. Willem van Hildegaersberch, un poète qui a vécu jusqu'à 1408 , a dédié un poème au roi du Portugal. Ce n'est pas tous les jours qu'on écrit des poèmes à ses ennemis. BOER, Dick E. H. de, « Looking at the Top of the Ant-hill. Some Observations. », STABEL, Peter, BLONDÉ, Bruno, GREVE, Anke (dir.), International Trade in the Low Countries (14 $16^{\text {th }}-16^{\text {th }}$ centuries), Leuven-Apeldoorn, Garant, 2000, p. 250.

66. BRAUDEL, Fernand, Civilização material, economia e capitalismo, séculos XV-XVIII. O jogos das trocas, vol. II, Lisboa, Teorema, 1992, p. 33.

\section{RÉSUMÉS}

La Flandre représente un marché très important pour les marchands portugais au Bas Moyen Âge. Ils disposaient d'un comptoir à Bruges et les ducs de Bourgogne leur avaient accordé une large liberté commerciale et le droit d'exercer la justice au sein de leur communauté. Pourtant, des conflits commerciaux et des intérêts politiques divergents donnant lieu à des violences eurent des répercutions fâcheuses sur l'activité des Portugais en Flandre. Ces conflits avaient-ils pour origine la nationalité des marchands ? Les Portugais étaient-ils considérés alors comme des étrangers et, de ce fait, privés de leurs droits? Quels mécanismes les protégeaient des tromperies, des vols et de la violence? Ce travail, élaboré à partir de sources flamandes, bourguignonnes et portugaises, constitue un premier essai au sein d'une recherche en cours. Il se propose de faire état des interrogations et d'apporter quelques éléments de réponse à partir des informations dont on dispose sur les activités des marchands portugais en Flandre à la fin du Moyen Âge.

Flanders has always been an important marketplace for Portuguese merchants, in the later Middle Ages. Freedom of trade was often granted by the dukes of Burgundy, ensuring the establishment of the Portuguese factory, and the right to apply justice within the community. Yet, commercial conflicts, political interests, and seldom cases of violence would have an effect on international trade. Would those conflicts be based on the nationality of merchants? Would Portuguese merchants be regarded as foreigners and so be deprived of their rights? Which mechanisms would have allowed them protection from cheating, theft and violence? This paper, sustained by Flemish, Burgundian, and Portuguese sources, being a first draft of an ongoing investigation, issues these questions while presenting some data on the activity of Portuguese merchants in medieval Flanders.

\section{INDEX}

Index chronologique : XVe siècle, XIVe siècle

Thèmes : Flandre 


\section{AUTEUR}

\section{FLÁVIO MIRANDA}

Doctorant Université de Porto - CITCEM boursier de la Fundação para a Ciência e a Tecnologia 